\title{
Relative Positioning of Vehicles Carrying Hazardous Materials Using Real-Time Kinematic GPS
}

\author{
Hee Sung Kim¹, Kwang Ho Choi', Je Young Lee', Joon Hoo Lim¹, Se Bum Chun², Hyung Keun Lee ${ }^{1 \dagger}$ \\ ${ }^{1}$ School of Electronics, Telecomm. \& Computer Eng., Korea Aerospace University, Goyang 412-791, Korea \\ ${ }^{2}$ Korea Aerospace Research Institute, Daejeon 305-806, Korea
}

\begin{abstract}
It is well known that the accident of vehicles carrying hazardous materials incurs huge losses economically and socially. To detect and respond rapidly against the accident of a vehicle carrying hazardous materials, it is essential to estimate the relative navigation information between the forward tractor module and the backward trailer module of the vehicle reliably and accurately. In this paper, a precise relative positioning system based on GPS is designed, implemented, and evaluated as a prerequisite to design an effective relative navigation system for the vehicle carrying hazardous materials. An experiment using field-collected $10 \mathrm{~Hz}$ real GPS measurements showed that the designed relative positioning system achieves $22 \mathrm{~cm}$ accuracy within 15 epochs by float solutions. Also, it was found that $\mathrm{cm}$-level integer solutions can be generated reliably after the quick convergence of float solutions.
\end{abstract}

Keywords: GPS, real-time Kinematic (RTK), moving reference, relative position

\section{INTRODUCTION}

The accident of vehicles carrying hazardous materials occurring in downtown area has a possibility of secondary damage due to the hazardous material spill even after the primary damage of accident, and therefore incurs huge losses in economic and social aspects considering the extent and spread of damage. For minimizing the loss incurred by the accident of vehicles carrying hazardous materials, it is necessary to quickly detect and take action against the accident of vehicles carrying hazardous materials.

A large vehicle carrying hazardous materials consists of tractor and trailer. To effectively and promptly detect the accident occurrence, the precise relative position, relative velocity, and attitude information between the tractor and trailer are required. In general, the GPS and INS are mainly used to obtain the position information and attitude

Received Apr 08, 2013 Revised Apr 28, 2013 Accepted Apr 29, 2013

tCorresponding Author

E-mail: hyknlee@kau.ac.kr

Tel: +82-2-300-0131 Fax: +82-2-3158-5769 information, and they complement each other by the integration of two different technologies (Hong et al. 2004) (Hong et al. 2005). The GPS has a disadvantage that the accuracy is degraded due to the effect of the error from the radio environment such as multipath or cycle slip, and has a limit in high-speed estimation of the state of tractor and trailer because it only provides the information of relatively low period. On the other hand, the INS has an advantage that it provides the information of high period and is almost not affected by the external environment, which improves the continuity of vehicle state estimation.

The GPS/INS integration technique improves the highspeed state estimation of vehicles or the continuity, but the accuracy of positioning largely depends on the performance of designed GPS positioning filter. Therefore, the precise absolute/relative positions of tractor and trailer become the basic technology for estimating the precise attitude information, and a GPS positioning technique which has high accuracy is required for this purpose. As for the GPS point positioning, the positioning accuracy is generally degraded due to the tropospheric delay, ionospheric delay, and satellite/receiver clock bias. The differential GPS (DGPS) 
was developed to effectively eliminate the error sources and improve the position accuracy, and when the DGPS is utilized, the satellite clock bias, tropospheric/ionospheric delay bias, and receiver clock bias can be effectively reduced or removed. As the DGPS, which only uses code measurements, has a measurement error of several meters, the Real-Time Kinematic (RTK) which simultaneously uses carrier phase measurements is frequently used for precise and accurate positioning. As for the RTK, the positioning accuracy of dozens of centimeters can be obtained even when it is in motion, and the accuracy of several centimeters can be achieved when the integer ambiguity is resolved (Hwang \& Brown 1990, Hwang et al. 1999).

Fig. 1 shows the schematic diagram of the system for estimating the state and attitude of vehicles carrying hazardous materials. In Fig. 1, the wireless data link is used to exchange the accident and state information, and the front payload estimates precise state and detects accident by receiving the GPS measurements from the rear payload.

In this study, the configuration of the system for estimating the precise absolute/relative positions of vehicles carrying hazardous materials is designed, and the relevant element algorithm is implemented. For generating a precise position, the integer ambiguity resolution is essential, and this is based on the performance of the filter for effectively estimating the float solution. In order to estimate the precise absolute/relative positions, a typical precise relative positioning method based on fixed reference station and a relative positioning technique based on moving reference station where the reference station is not fixed are required, and the position domain filter based on moving reference station is implemented for this purpose.

The simulation and actual vehicle onboard experiment were conducted to analyze the performance of the designed system. The performance of implemented filter is analyzed in terms of accuracy, and the performance of ambiguity resolution is analyzed with the Time To Ambiguity Fix (TTAF) (Feng \& Wang 2008).

\section{A SYSTEM FOR ESTIMATING THE STATE OF VEHICLES CARRYING HAZARDOUS MATERIALS}

For detecting the accident occurrence of vehicles carrying hazardous materials, the absolute position of vehicle and the precise relative position, relative velocity, and attitude information between the tractor and trailer are required. To estimate the precise absolute/relative positions, relative velocity, and attitude information, the navigation technique

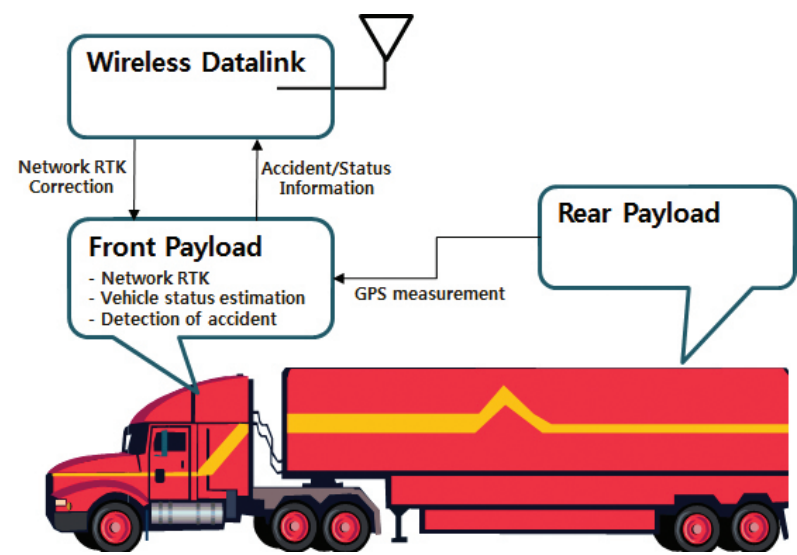

Fig. 1. Configuration of navigation system for vehicles carrying hazardous materials.

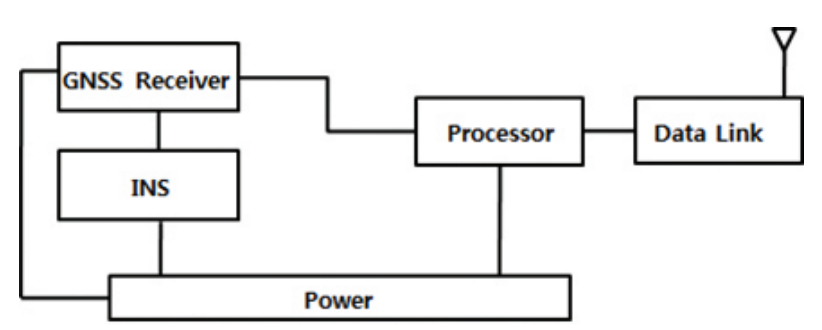

Fig. 2. Composition of front/rear payload.

which only uses the GPS and the navigation technique which uses the GPS and INS are typically utilized.

Fig. 2 shows the schematic diagram of the front/rear payloads of vehicles carrying hazardous materials. The GNSS receiver and INS transfer the measured data to the processor. The data link uses the Ethernet or RF modem, and is used for transmitting the data link between the front and rear payloads and the estimated vehicle state information to the control center. The processor estimates the relative position/velocity between the front and rear and the attitude using the data received from the GNSS and INS.

In general, the steady-state position accuracy of GPS/ INS integrated navigation system largely depends on the GPS position accuracy which is used as the auxiliary measurement. Though the navigation technique which only uses the GPS has a disadvantage of providing only the information of relatively slow output rate, it is an essential technological element for estimating the precise absolute/ relative positions which are the basis of state estimation. Therefore, for the final development of GPS/INS relative navigation system shown in Fig. 2, as the preliminary research, the relative positioning system which only uses the GPS needs to be designed and the accuracy of the system needs to be analyzed. For this purpose, a system for estimating the precise relative position was designed 


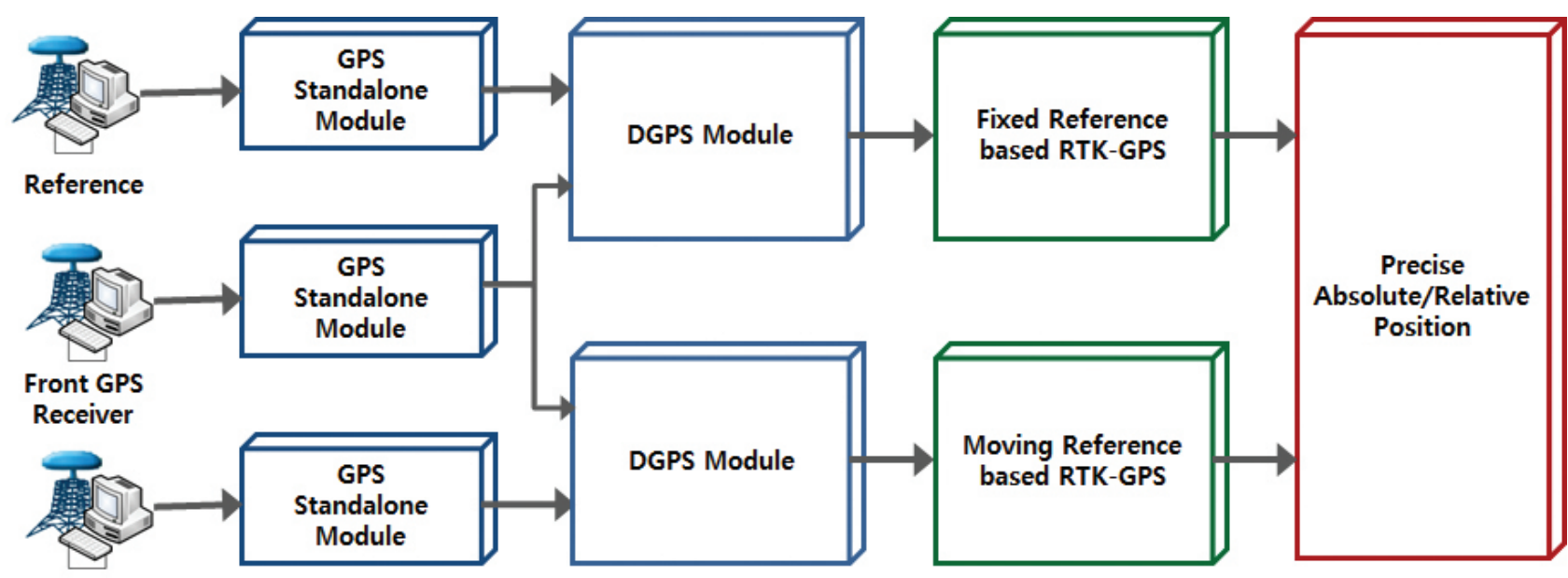

Rear GPS

Receiver

Fig. 3. Data processing architecture of designed system.

in this study as shown in Fig. 3 by installing GPS receivers at the fixed position and the tractor and trailer of vehicle carrying hazardous materials, respectively. In Fig. 3, the reference and front GPS data are integrated by the DGPS module, and select the valid satellites through the processes of measurement differencing, malfunction detection, and satellite position calculation. The selected measurement is used for generating the absolute position of tractor in the fixed reference based RTK-GPS module.

The rear GPS data is integrated by the front GPS data and DGPS module, and is used as the input of moving reference based RTK-GPS module through the valid satellites selection process. The fixed/moving reference based RTKGPS modules perform the integer ambiguity search based on the float solution generated from the implemented filter, and estimate the precise absolute/relative positions.

\section{IMPLEMENTATION OF CORE TECHNOLOGY}

\subsection{Double difference position domain Hatch filter}

The signal precision of GPS is degraded due to various error sources. These error sources include the satellite clock bias, tropospheric/ionospheric delay bias, and receiver clock bias, and they can be reduced or removed by the single/double differencing technique (Parkinson \& Axelad 1996). This study uses double differenced measurements, and when the distance between the two receivers is assumed to be less than several kilometers, the code/carrier phase measurement equation can be modeled as Eq. (1).

$$
\begin{aligned}
& \tilde{\boldsymbol{\rho}}_{k}^{j o}=\left(e_{k}^{j o}\right)^{T}\left[X,-X_{u, k}\right]+V_{k}^{j o} \\
& \tilde{\varphi}_{k}^{j o}=\left(e_{k}^{i o}\right)^{T}\left[X_{,}-X_{U, k}\right]+\lambda N_{k}^{j o}+n_{k}^{j o}
\end{aligned}
$$

where $e_{k}^{j o}$ is the line-of-sight angle vector, $X_{r}$ and $X_{u, k}$ are the positions of reference and rover, respectively, $v_{k}^{j o}$ and $n_{k}^{j c}$ are the measurement errors of code and carrier phase, respectively, $\lambda$ is the wavelength, and $N_{k}^{j o}$ is the integer ambiguity. Also, in Eq. (1), the subscript $\mathrm{k}$ indicates the observation epoch, and $\mathrm{r}$ and $\mathrm{u}$ indicate the reference and rover, respectively. The superscript $\mathrm{j}$ and $\mathrm{o}$ indicate the satellite number, and o indicates the reference satellite.

An efficient navigation filter should provide the accuracy of estimated position information and the relevant index sufficiently. In this regard, most navigation filters calculate the error covariance matrix which represents the statistical characteristics of estimation error, along with the position estimates. The validity of error covariance matrix that the filter calculates with respect to the navigation error that actually occurred has a large effect on the navigation performance (Lee 2005).

The range domain Hatch filter prevents the deterioration of position accuracy due to the inaccurate kinematic model by sufficiently utilizing the diversity of receiver measurement, and is used for various applications of geodetic survey and aviation fields by various combinations of dual-frequency measurement (Hatch 1982).

For the range domain Hatch filter, there is a risk that the losses of position accuracy and error covariance data become large when the number of visible/valid satellites changes. To overcome the performance degradation of range domain Hatch filter depending on the change of 
Table1. Double difference position domain Hatch filter.

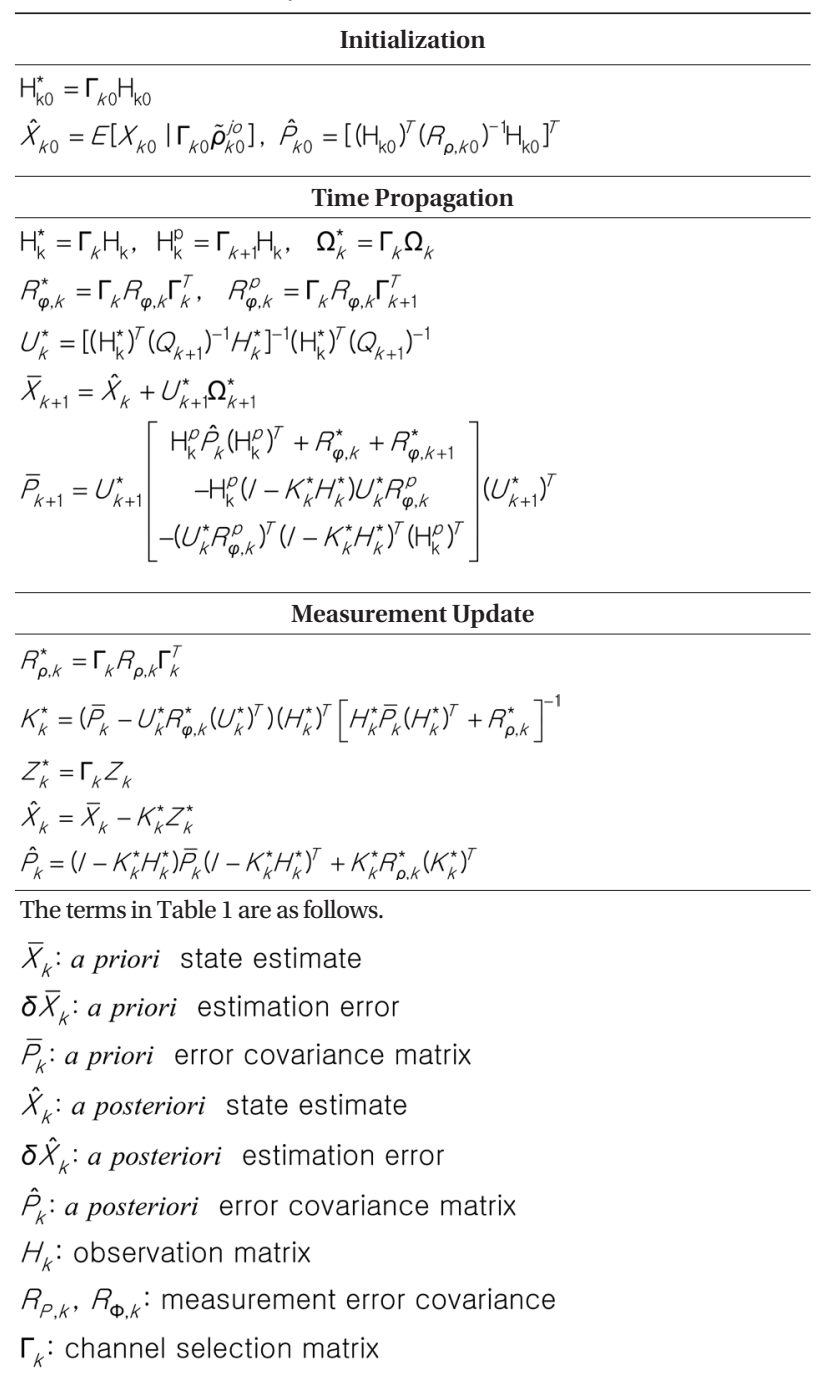

satellite combination, the position domain Hatch filter has been introduced and shown that stable generation of float solution and error covariance data is possible despite the change of satellite combination (Lee \& Rizos 2008, Kim \& Lee 2008).

The double difference position domain Hatch filter is robust to satellite slip or the change in the number of satellites, and improves the reliable performance of ambiguity resolution through the generation of consistent and realistic error covariance data. Also, it is advantageous for the high-speed relative positioning by minimizing the estimation errors. Thus, the double difference position domain Hatch filter is used in this study to estimate the absolute/relative positions of vehicles carrying hazardous materials. Table 1 shows the double difference position domain Hatch filter.

The channel selection matrix $\Gamma_{k}$ selects only the valid satellite channels at each epoch, and is used to consider the change of satellite combination and the change of reference satellite between the previous and current epochs. The indirect measurements $Z_{k}$ and $\Omega_{k+}$, which are used for time propagation and measurement update, are shown in Eqs. (2) and (3), respectively.

$$
\begin{aligned}
Z_{k} & =H_{k} \bar{X}_{k}-Y_{k} \\
& =H_{k} \delta \bar{X}_{k}+v_{k} \\
\Omega_{k+1} & =-\Delta H_{k} \hat{X}_{k}-\left(\Phi_{k+1}-\Phi_{k}\right)
\end{aligned}
$$

\subsection{Relative positioning technique based on moving reference}

For a typical RTK-GPS technique, the reference position $X_{r}$ is located at a fixed, known position. Using this reference, a rover can estimate the absolute position by generating or receiving the correction data. However, for the moving reference based RTK-GPS technique, the reference position moves along with the position of rover, and the baseline vector between the two rovers is estimated using a direct differencing method rather than a method generating the correction data. Also, in Eq. (1), the term for the reference station $X_{r}$ is changed to $X_{r, k}$.

In terms of time synchronization, the fixed reference based RTK-GPS can maintain accuracy for a certain period of time even when there is a loss of reference data. However, the moving reference based RTK-GPS can accurately estimate the baseline only when accurate time synchronization is achieved between the two receivers. Fig. 4 shows the error sources which can be caused by asynchronous time in the moving reference based RTK-GPS. The moving reference based RTK-GPS estimates the relative receiver position in relation to the reference receiver, and when the time synchronization is not accurately achieved, the error occurs in the baseline vector estimation between the two rovers which have different movements. This error becomes larger as the two rovers move faster. Therefore, to reduce the error caused by the asynchronous time between

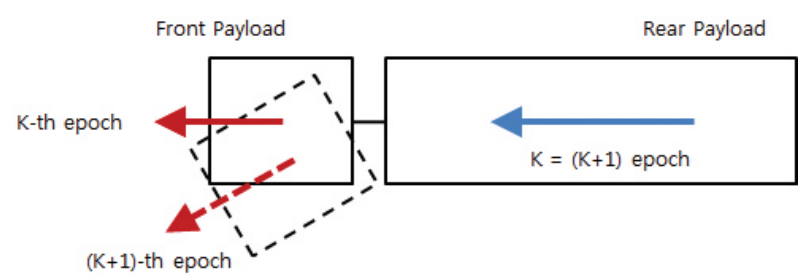

Fig. 4. RTK-GPS error caused by asynchronous clock in moving reference based RTK-GPS 


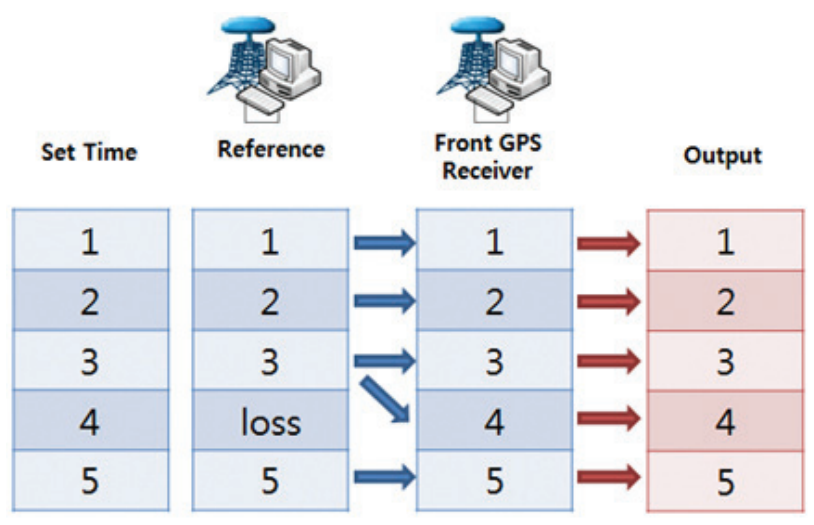

(a) Fixed Reference Based RTK-GPS

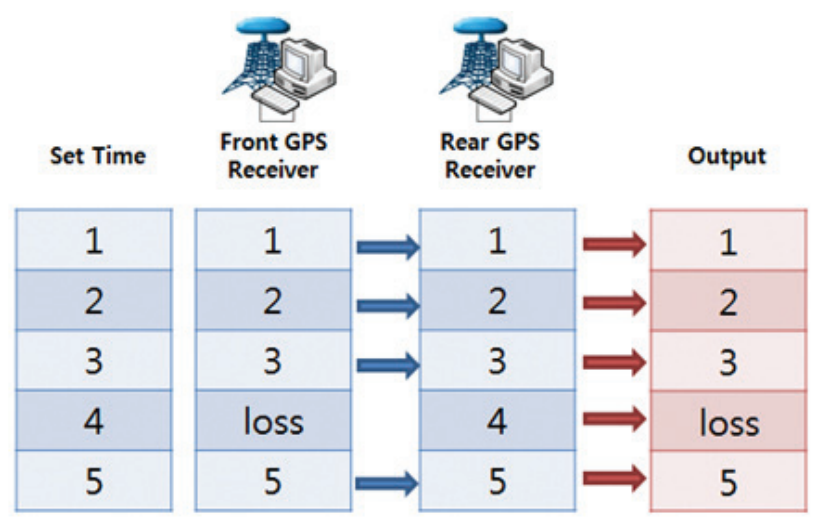

(b) Moving Reference Based RTK-GPS

Fig. 5. Effects of data loss on solution output in fixed/moving reference based RTK-GPS.

the two receivers, the time between the two receivers needs to be matched using the 1PPS signal in terms of hardware, and the data set received from the two receivers needs to be matched or preparation for the data loss is required in terms of software. Fig. 5 shows the effect of the data loss of fixed/moving reference based RTK-GPS on the output of navigation solution. For the fixed reference based RTKGPS, the generation of navigation solution is possible using the reference receiver data of previous epoch even when there is a data loss in the reference receiver data. However, for the moving reference based RTK-GPS, the generation of navigation solution is not possible when there is a data loss in either of the two receivers.

Unlike the fixed reference based RTK-GPS, the major characteristic of moving reference based RTK-GPS is that the baseline between the two receivers fixed at adjacent rovers is several meters to dozens of meters, which is very short. In the relative position determination, when compared to the fixed reference based RTK-GPS, the moving reference based RTK-GPS technique can more effectively eliminate the atmospheric error component such as tropospheric/ ionospheric delay bias depending on baseline length, which is considerably advantageous for the generation of precise float solution and the ambiguity resolution.

The variables related with the data processing for stable float solution and ambiguity resolution are as follows.

i) Elevation mask: 10 degree

ii) Positioning mode: Moving

iii) Measurements used: L1 pseudorange/carrier phase

iv) Use the Doppler data

v) Threshold (ratio test): 3

For prompt generation of integer solution, the float solution is first estimated by the double difference position domain Hatch filter, and based on this, the integer solution is generated by an ambiguity resolution module. The Least square AMBiguity Decorrelation Adjustment (LAMBDA) technique is used for the generation of integer solution (Teunissen 1994). The LAMBDA technique has an advantage of shortening unnecessary search time by decorrelating the generated float solution and error covariance data, which is suitable for the high-speed precise absolute/relative position estimation. The validity of generated integer ambiguity is judged by the integer solution generation module. The ratio test and residual test methods are used for this purpose, and the generated integer ambiguity is regarded as the reliable integer ambiguity only when it passes both of the tests.

\subsection{Front/Rear payload and data transfer protocol}

Fig. 6 shows the exterior of front/rear payload, and the interior of the equipment is loaded with the NovAtel ProPak-V3 GPS dual-frequency receiver, NovAtel UIMU-H58 IMU equipment, and NovAtel GPS-703-GGG antenna. The payload was designed so that it can be linked with a simulator, and the front/rear payload can transmit/receive the data through Ethernet.

The data processor receives the channelwisemeasurements and navigation data for each satellite from the GPS receiver by the protocol of NovAtel Company (NovAtel 2010). The channelwise-measurements include

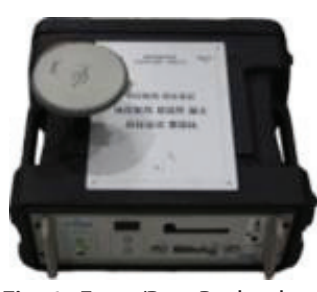

Fig. 6. Front/Rear Payload. 
Table 2. Comparison of data size w.r.t NovAtel and RTCM 3.0 protocols.

\begin{tabular}{ccc}
\hline (number of satellites: 10) & $\begin{array}{c}\text { NovAtel } \\
\text { ID: Range }\end{array}$ & $\begin{array}{c}\text { RTCM v3.0 } \\
\text { ID: 1004 }\end{array}$ \\
\hline Data Size (Bytes) & 470 & 164 \\
\hline
\end{tabular}

various measurements such as pseudorange, carrier phase, Doppler, and signal strength, and the navigation data includes the parameters for determining the orbit of each satellite.

The measurement received from the rear payload is transferred to the front payload for processing. As for the measurement outputted at slow rate, the data delay is not a big problem though the NovAtel protocol is used. However, if the output of measurement is at a high rate of more than $10 \mathrm{~Hz}$, a problem arises in transmitting the data to the front payload using the NovAtel protocol. The data delay not only causes the delay of data processing, but also increases the possibility of data loss. Therefore, a more efficient message protocol is required which has a smaller data size and includes the measurements necessary for generating the navigation solution. The standardized message protocols such as RTCM and CMR are widely used for the real-time GPS/GNSS data transfer, and in this study, the measurement from the rear payload is transferred to the front payload using the 1004 messages among the RTCM v3.0 (RTCM 2006).

Table 2 compares the sizes of L1/L2 measurements for 10 satellites between the RANGE data among the NovAtel

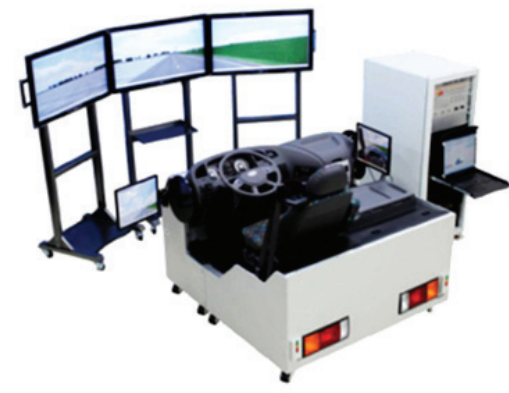

(a)

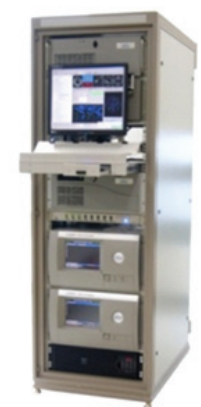

(b)

Fig. 7. (a) Simulator for generating vehicle trajectory, (b) GPS signal generator.

protocol and the RTCM v3.0 1004 data. As shown in Table 2 , when the RTCM v3.0 protocol is used, the data size is reduced by about one-fourth.

\section{SIMULATION \& EXPERIMENT}

A simulation was performed to investigate whether the designed system can precisely estimate the relative position of vehicles carrying hazardous materials, and an actual vehicle experiment was performed to analyze the estimation performance for the absolute/relative positions of vehicle.

The vehicle simulator and GPS signal generator (Spirent) shown in Fig. 7 were used to generate the simulation data. The vehicle simulator generates the trajectory of vehicle,

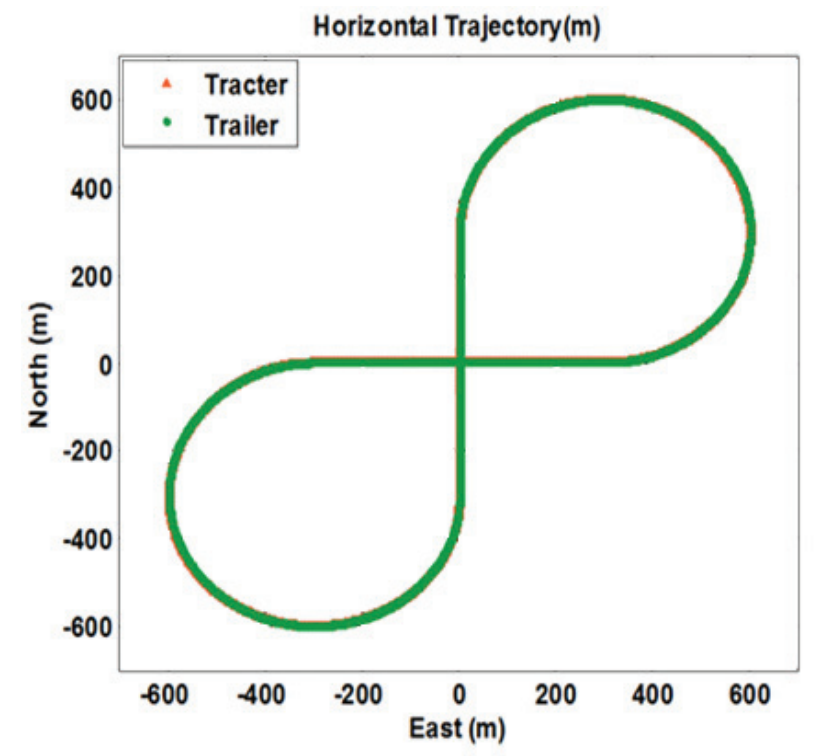

(a)

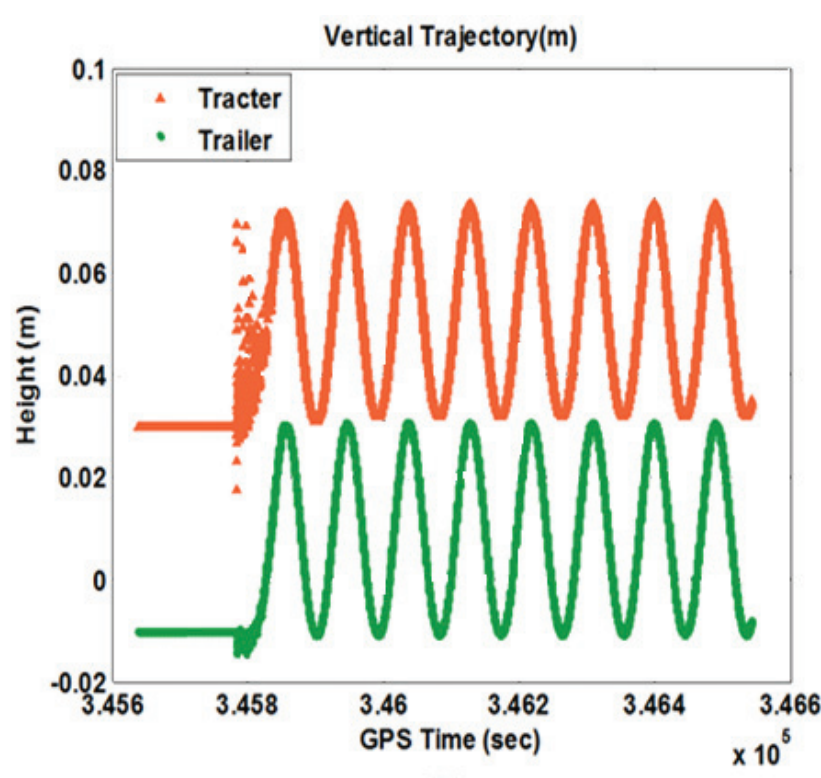

(b)

Fig. 8. Horizontal and vertical trajectories of vehicle in simulation. 


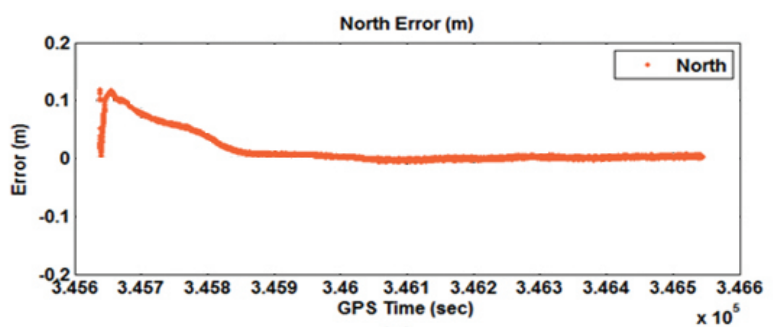

(a)

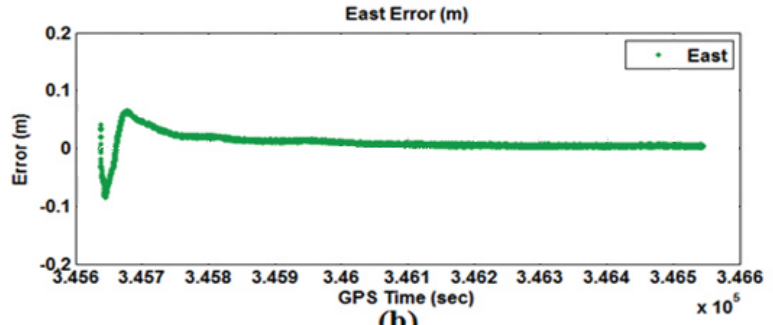

(b)

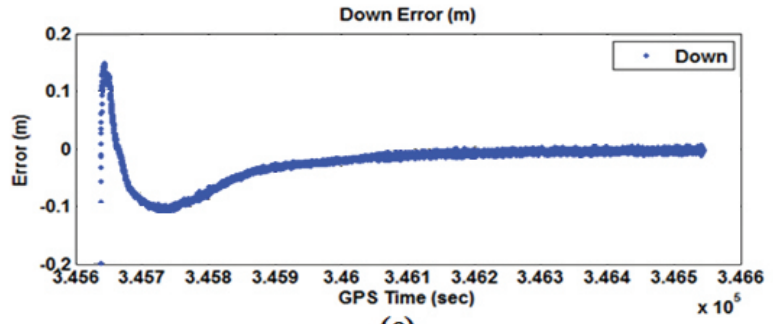

(c)

Fig. 9. Results of float solution (baseline vector).

and the signal generator generates the GPS signal using the vehicle trajectory.

The simulation was designed to be similar to the actual condition, and the baseline of front and rear vehicles was made to be $4.145 \mathrm{~m}$. The GPS signal is generated with a frequency of $10 \mathrm{~Hz}$. When the generated signal is transferred to the front and rear payloads, the rear payload transmits the received GPS data to the front payload in real time, and the data processor at the front payload generates the navigation solution in real time.

Fig. 8 shows the simulation horizontal and vertical trajectories generated from the simulator, and it completes the trajectory of figure of eight three and a half times.

Fig. 9 shows the error tendency relative to reference trajectory for the float solution generated from the designed system. As shown in Fig. 9, the float solution is generated with an error value of less than $10 \mathrm{~cm}$ relative to reference trajectory. Fig. 10 shows the baseline estimation error of float solution (a) and the baseline estimation result relative to reference trajectory (b). In the entire experiment section, the error was found to be less than $5 \mathrm{~cm}$.

Figs. 11 and 12 show the baseline vector estimation error tendency of integer solution and the baseline estimation error of integer solution, respectively. The integer solution based on the float solution was generated simultaneously with the initiation of filter, and the NED direction and baseline estimation errors were found to be less than $5 \mathrm{~mm}$, which is very precise. The positioning performance of float and integer solution is summarized in Table 3.

As the simulation cannot perfectly consider the actual condition, an experiment in the actual environment is required to analyze the performance of the designed system. A large vehicle which consists of tractor and semitrailer was used for the experiment, and the payload of Fig. 6 was installed at the front and the rear. In order to evaluate the positioning performance of fixed reference based RTKGPS, unlike the simulation, the experiment was performed

Table 3. Performance of float and integer solution w.r.t. simulation.

\begin{tabular}{ccc}
\hline & Float & Integer \\
\hline 2D RMS error $(\mathrm{m})$ & 0.037 & 0.0015 \\
3D RMS error $(\mathrm{m})$ & 0.057 & 0.0027 \\
\hline
\end{tabular}
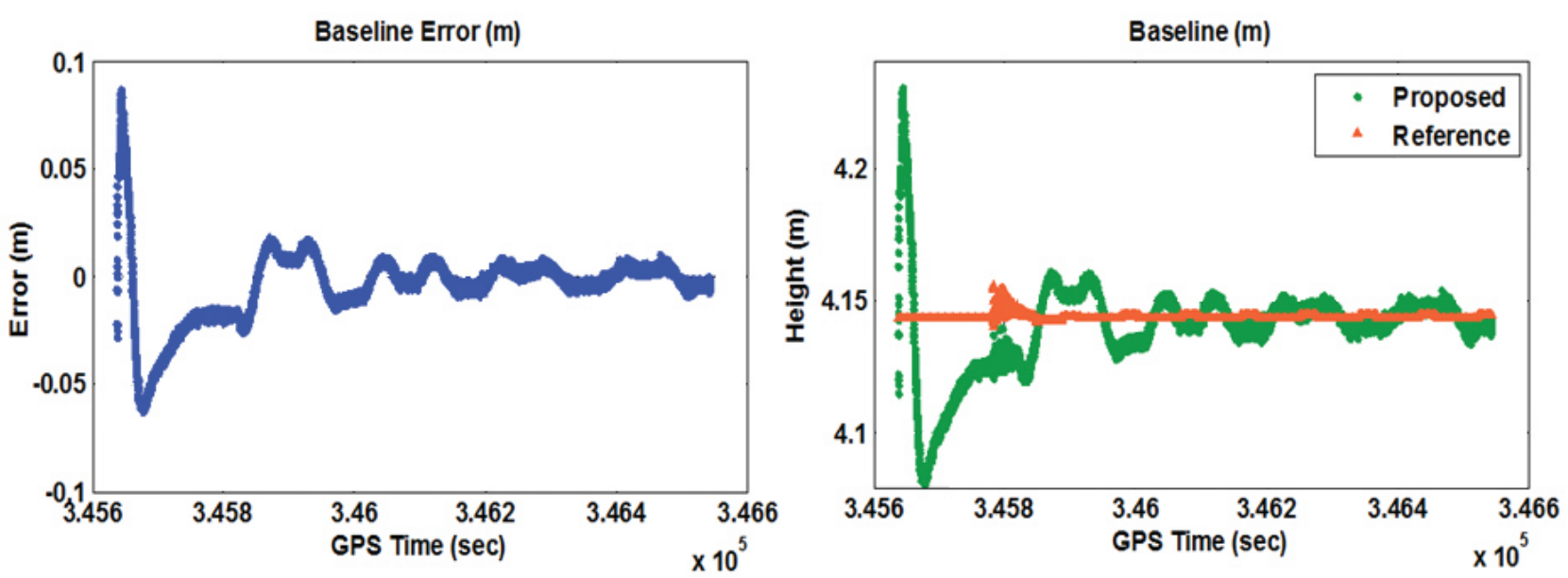

Fig. 10. Results of float solution (baseline). 


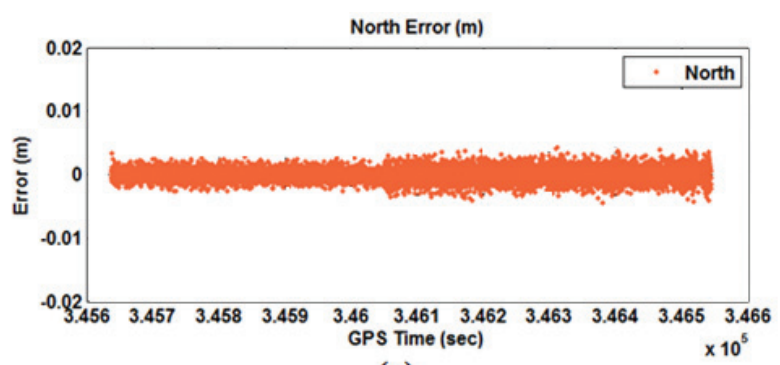

(a)

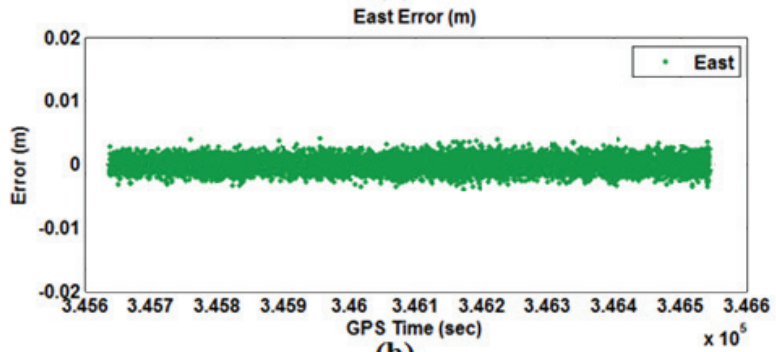

(b)

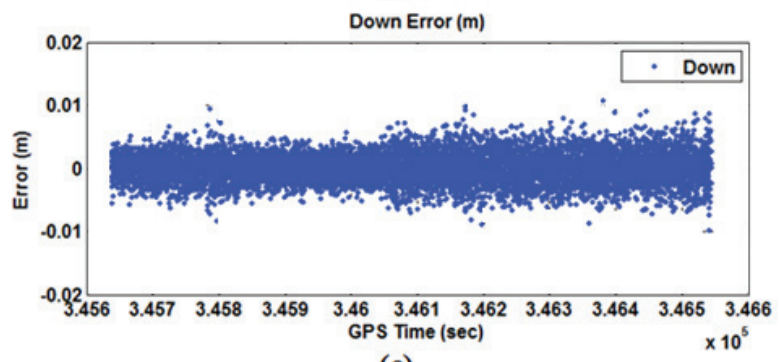

(c)

Fig. 11. Results of integer solution (baseline vector).

by installing a reference station in the vicinity of the experiment area.

The NovAtel DL-V3 was used as the reference receiver, which is capable of dual-frequency reception and multipleGNSS signal reception. The data rate of vehicle onboard receiver and reference receiver was made to be $5 \mathrm{~Hz}$, and the experiment was conducted for about 16 minutes.

The experiment was performed at the Korea Automobile Testing \& Research Institute of Korea Transportation Safety Authority which is located in Hwaseong, Gyeonggi Province, and the baseline of the installed reference station and the vehicle experiment area is $40 \sim 300 \mathrm{~m}$. Figs. 13 and 14 show the configuration of experiment and the experiment area, respectively.

Prior to processing the experiment data, the coordinate of the installed reference station needs to be determined. The reference station coordinate was determined using the reference receiver and the Suwon reference station data from the National Geographic Information Institute. The determined reference station coordinate is as follows.

ECEF-X: -3042975.9547 ECEF-Y: 4072334.2079 ECEF-Z: 3839020.7211

Fig. 15 shows the architecture of GPS data processing. The GPS data collected from the front/rear payloads and reference station is inputted as the Receiver Independent Exchange Format (RINEX). For the fixed reference based RTK-GPS experiment, the precise position of vehicle is estimated using the front/rear and reference GPS data. For the moving reference based RTK-GPS experiment, the baseline vector between the two receivers is estimated using only the front/rear GPS data.

For a reliable integer solution generation, the integer ambiguity which passed both the ratio test and residual test for 5 epochs is considered to be the integer ambiguity candidate, and when the integer ambiguity belongs to the integer ambiguity candidate for 5 consecutive epochs, it is regarded as the reliable integer ambiguity.

Fig. 16 shows the performance of ambiguity resolution
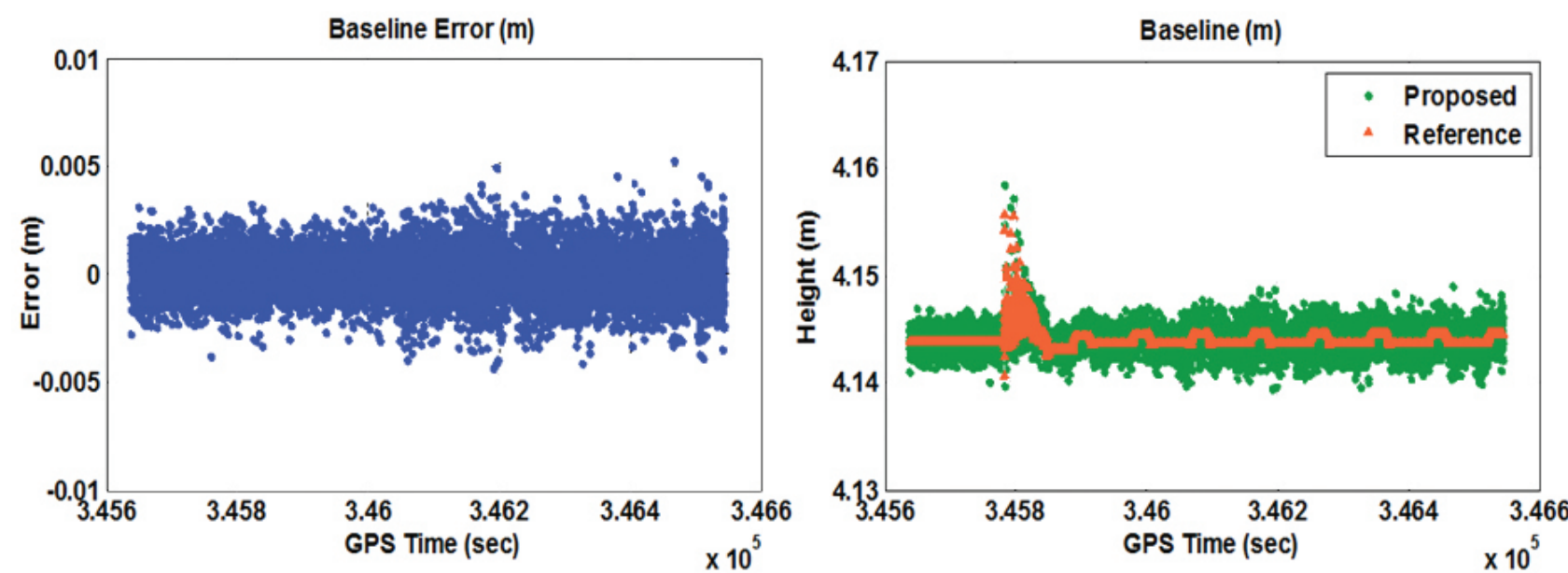

Fig. 12. Results of integer solution (baseline). 


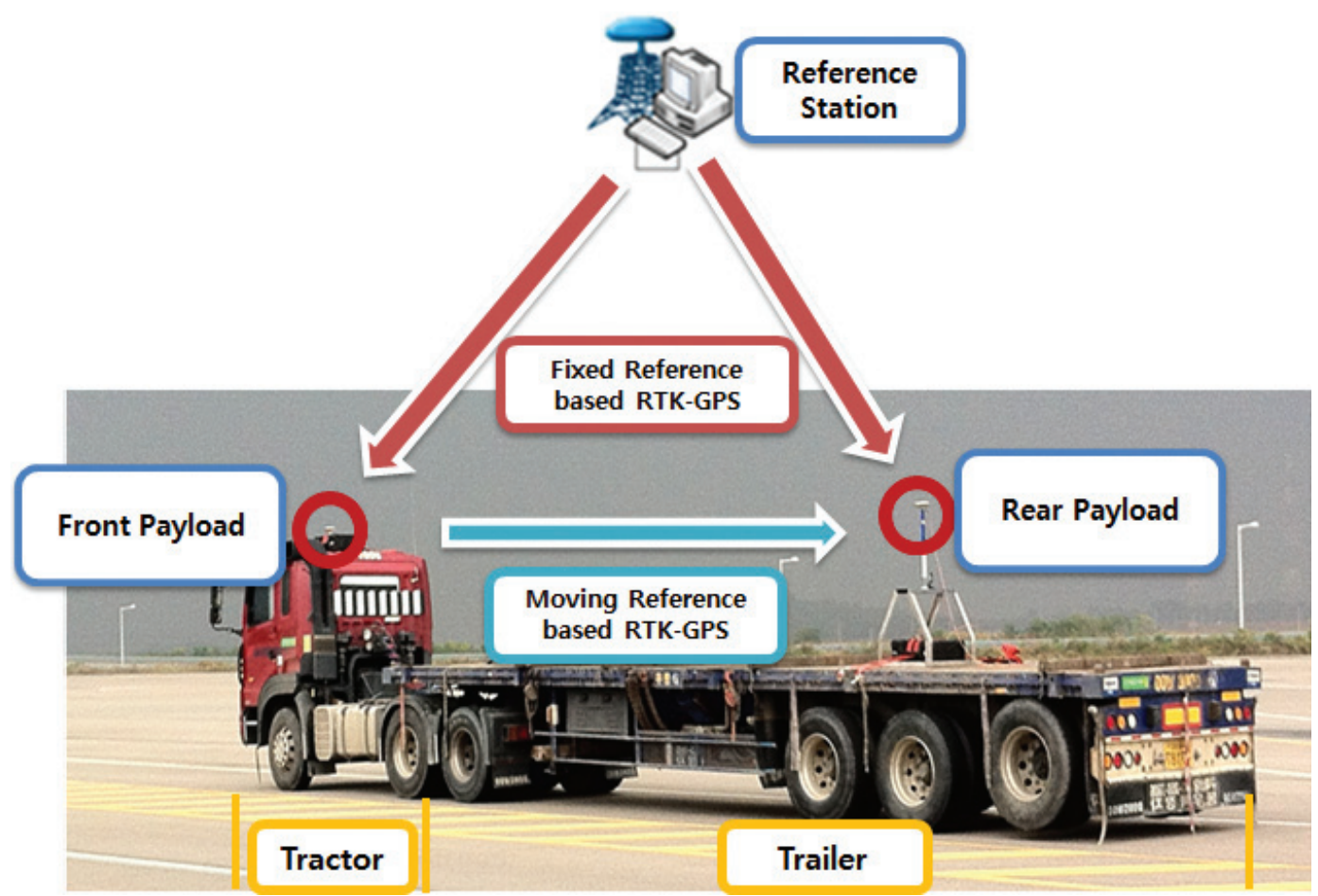

Fig. 13. Configuration of experiment.

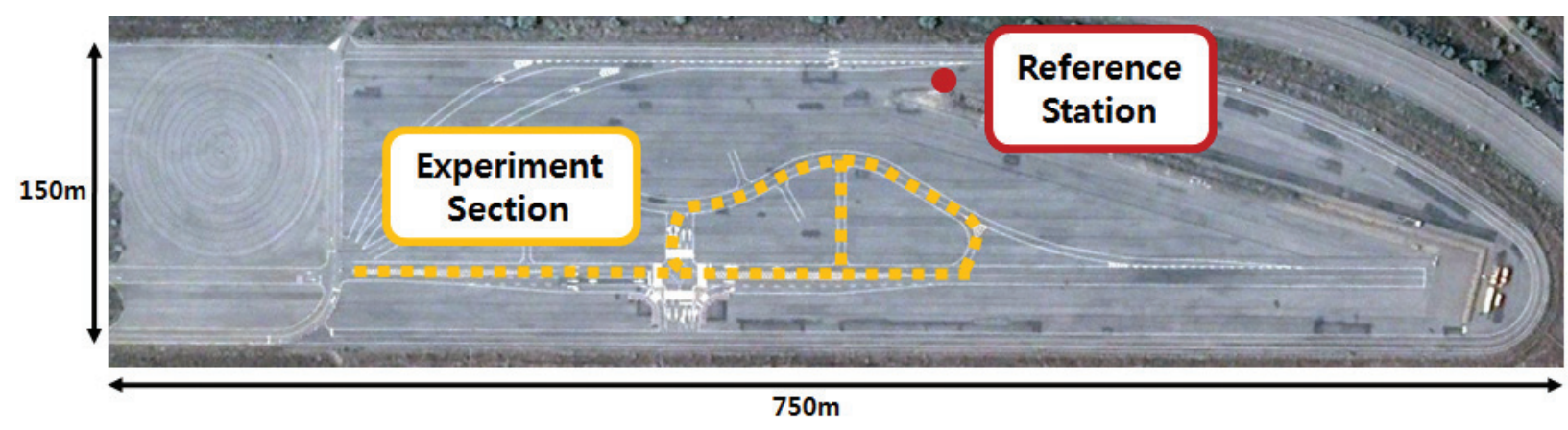

Fig. 14. Experiment area.

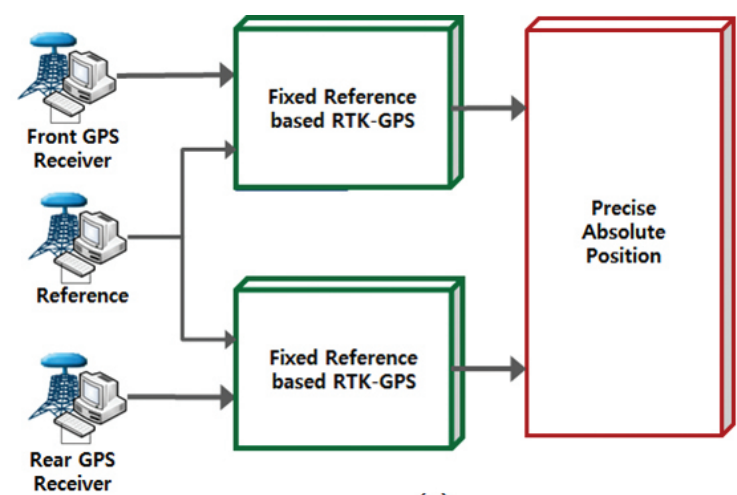

(a)

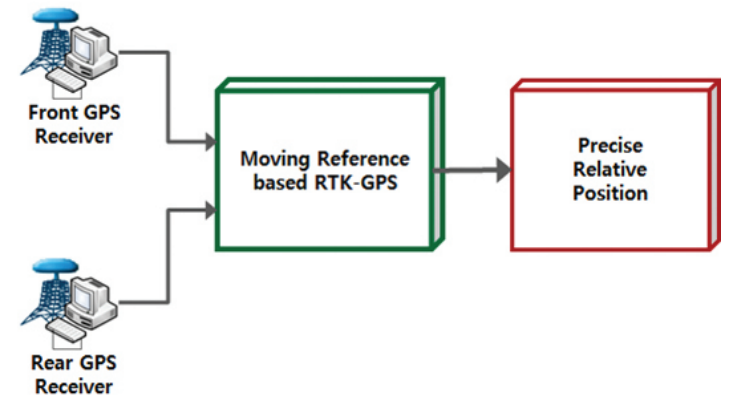

(b)

Fig. 15. Data processing architectures for (a) fixed reference based RTK-GPS, (b) moving reference based RTK-GPS. 


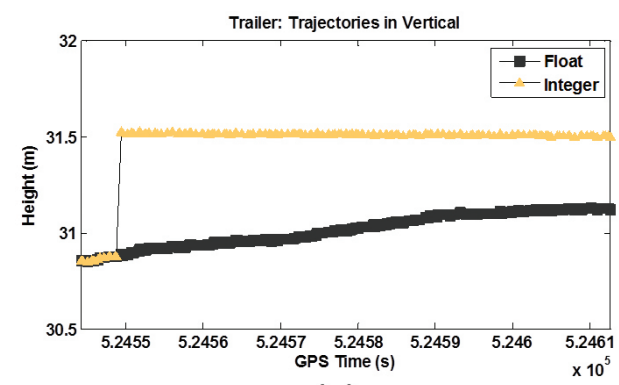

(a)

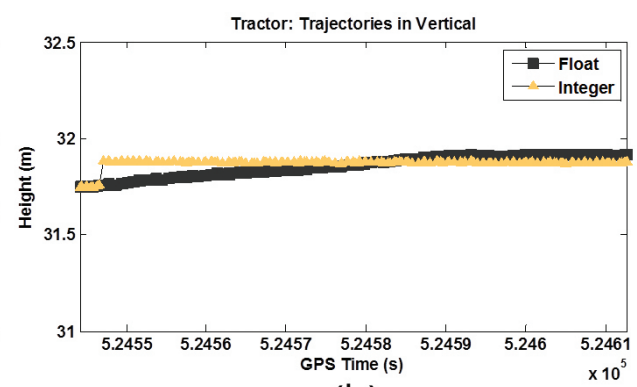

(b)

Fig. 16. Results of ambiguity resolution in the case of fixed reference based RTK-GPS (a) trailer, (b) tractor.

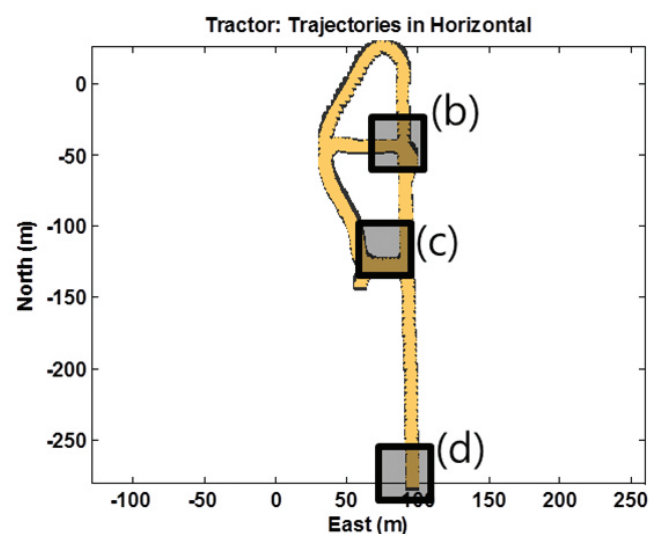

(a)

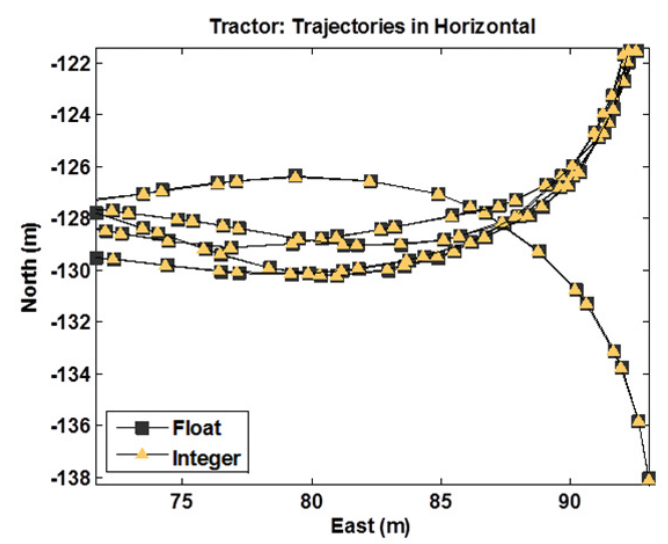

(c)

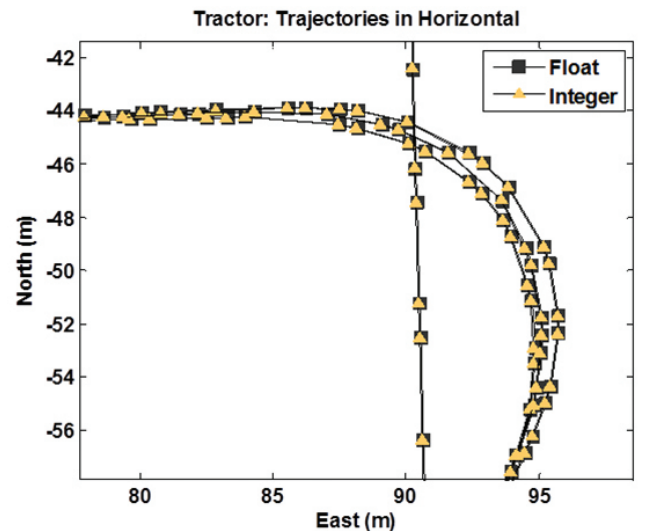

(b)

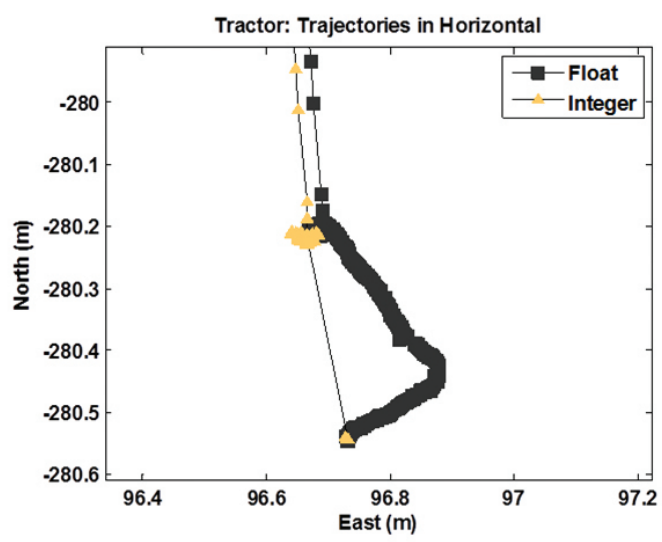

(d)

Fig. 17. Horizontal results of tractor (fixed reference).

Table 4. Performance of ambiguity resolution w.r.t. experiment.

\begin{tabular}{ccc}
\hline & Trailer & Tractor \\
\hline TTAF & $6.6(\mathrm{sec}): 17$ epochs & $4.2(\mathrm{sec}): 21$ epochs \\
\hline
\end{tabular}

for the fixed reference based RTK-GPS. Fig. 16a shows the performance of ambiguity resolution for the trailer onboard receiver data, and Fig. 16b shows the performance of ambiguity resolution for the tractor onboard receiver data.
As shown in Table 4, the ambiguity resolution was possible within 20 epochs for both receivers. In Table 4, regarding the trailer, the time needed for the initial ambiguity resolution is different from the number of epochs of the data, and this is thought to be caused by the data loss during data collection. When the data is collected in $5 \mathrm{~Hz}$, the data of total 33 epochs need to be received for 6.6 seconds, but the data of only 17 epochs were processed due to the data loss.

Figs. 17 and 18 show the horizontal positioning results 


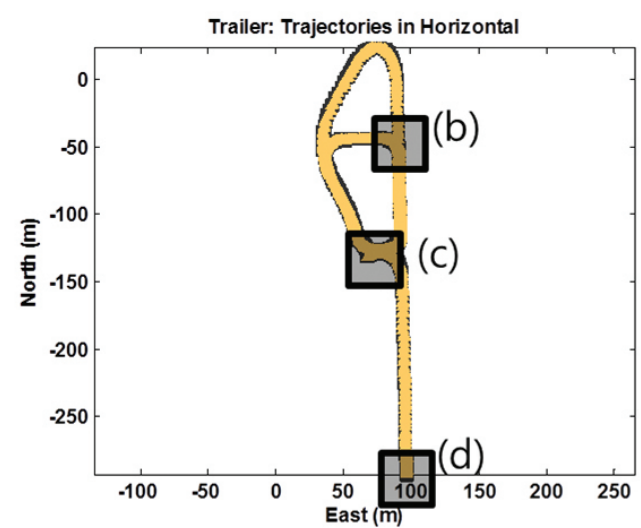

(a)

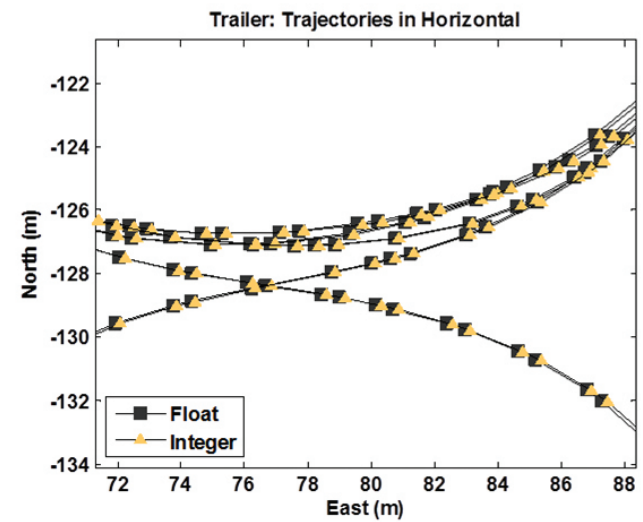

(c)

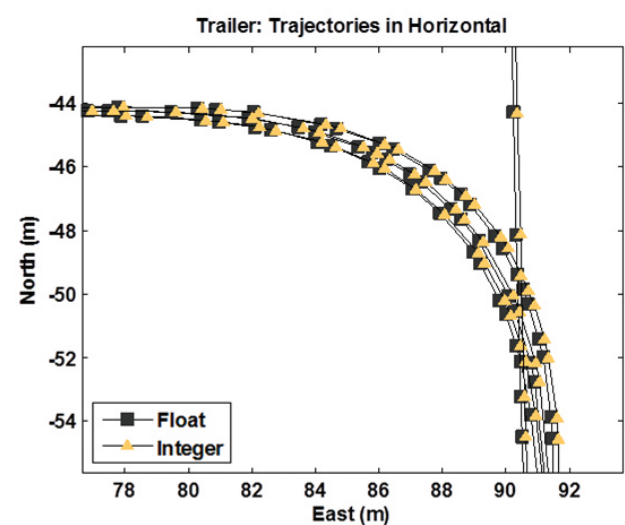

(b)

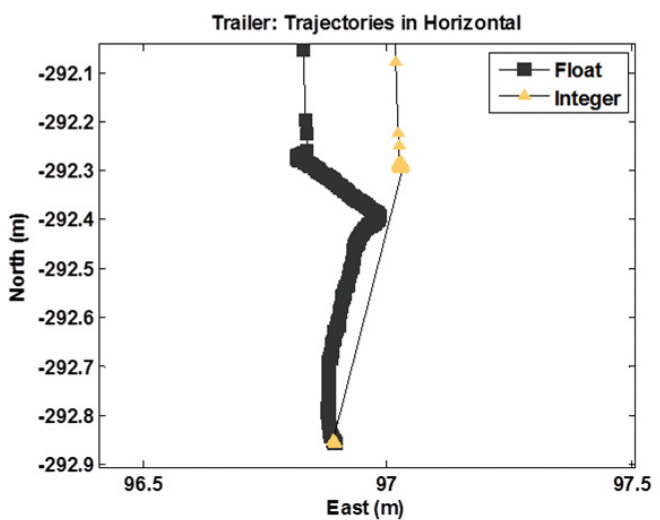

(d)

Fig. 18. Horizontal results of trailer (fixed reference).

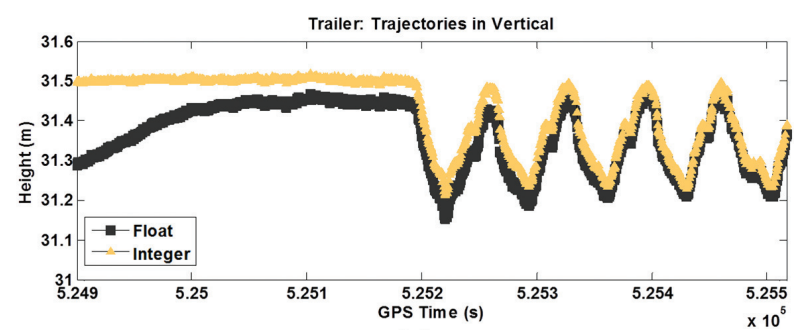

(a)

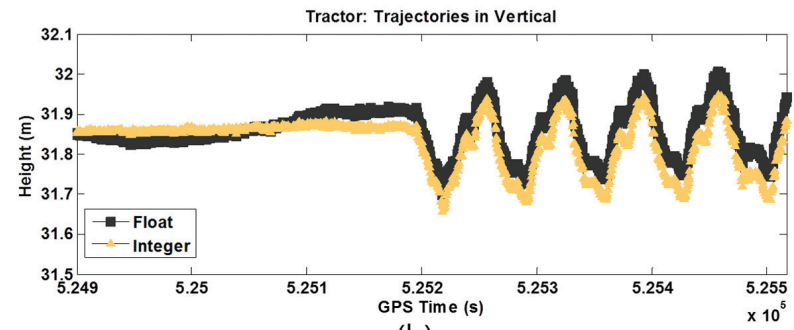

(b)

Fig. 19. Vertical results of (a) tractor, (b) trailer (fixed reference).

generated using the tractor and trailer data, respectively. In
Figs. 17 and 18, (a) shows the trajectory of vehicle, (b) and (c) show the turning sections of vehicle, and (d) shows the static section. Figs. 19a, b show the vertical trajectories of tractor and trailer, respectively.

For the tractor, after the initial transient period, the position was generated having the value that is within 10 $\mathrm{cm}$ of the result of integer solution. On the other hand, for the trailer, the convergence time was rather long during the initial transient period. This is thought to be the suboptimality problem which is caused by the use of smoothed measurement. When the filter converged, the error was found to be less than about $15 \mathrm{~cm}$. Table 5 shows the quantitative performance of float solution for the fixed reference based RTK-GPS experiment.

Fig. 20 shows the result of baseline estimation for the moving reference based RTK-GPS experiment. The data of 15 epochs was necessary for the initial ambiguity resolution time, and the stable integer ambiguity was generated in the entire experiment section. As shown in Table 6, the estimated 3D RMS error for the baseline vector of float solution with 
Table 5. Performance of float and integer solution w.r.t. simulation.

\begin{tabular}{ccc}
\hline & Tractor & Trailer \\
\hline 2D RMS error $(\mathrm{m})$ & 0.14 & 0.20 \\
3D RMS error $(\mathrm{m})$ & 0.14 & 0.30 \\
\hline
\end{tabular}

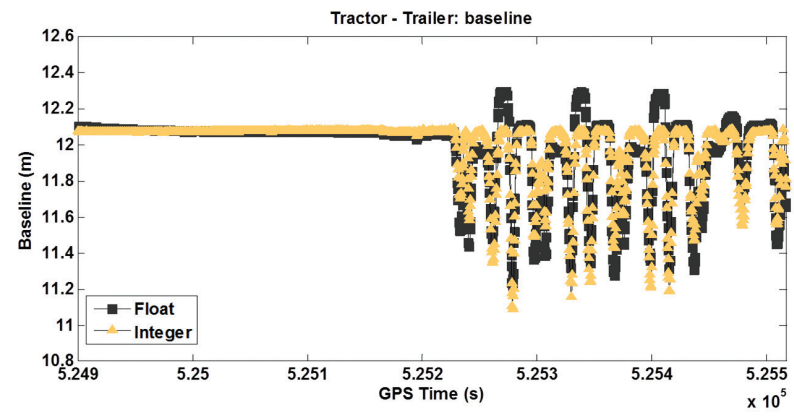

Fig. 20. Result of baseline estimation (moving reference).

Table 6. Results of baseline vector estimation (moving reference)

\begin{tabular}{ccc}
\hline & Static Section & Kinematic Sector \\
\hline 3D RMS error $(\mathrm{m})$ & 0.35 & 0.22 \\
\hline
\end{tabular}

respect to integer solution was $35.1 \mathrm{~cm}$ in the static section, and the result of baseline vector estimation based on moving reference also had a long convergence time of filter due to the sub-optimality problem of filter. After the convergence, the 3D RMS error of $22.2 \mathrm{~cm}$ was generated in the kinematic section. Also, the baseline estimation RMS error of about 8 $\mathrm{cm}$ was generated, which is a very satisfactory result. When the tractor and trailer stand in line, the maximum value of baseline was about $12.1 \mathrm{~m}$, and when the space between the tractor and trailer decreases in the turning section, the baseline decreased to a minimum of about $11.1 \mathrm{~m}$.

\section{CONCLUDING REMARKS}

In this study, a system was designed which estimates the precise absolute/relative position of vehicles carrying hazardous materials using the GPS, and the element technology was implemented using the double difference position domain Hatch filter. A simulation and an experiment using an actual large vehicle were performed to analyze the performance of the implemented element technology. In the simulation, the real-time positioning performance of the implemented system was analyzed using the reference trajectory, and the 3D RMS error of about $6 \mathrm{~cm}$ was generated for the float solution and that of about $3 \mathrm{~mm}$ was generated for the integer solution. In the experiment, there is a difficulty in generating the reference trajectory, and for analyzing the accuracy of float solution, the integer solution was generated with a post-processing method, which was then used as a true value. Through the experiment, the 3D RMS error of float solution with respect to integer solution was found to be $10 \sim 30 \mathrm{~cm}$ for the fixed reference based RTK-GPS, and the RMS error of float solution was found to be less than $10 \mathrm{~cm}$ for the moving reference based RTK-GPS. The performance of integer solution was analyzed using the initial fixed time, and the data of 20 epochs based on fixed reference and the data of 15 epochs based on moving reference were used for the generation of initial integer solution. For the stable generation of precise relative position, it is essential to utilize the integer solution which can be generated after the ambiguity resolution.

\section{ACKNOWLEDGMENTS}

This research was supported by Korea Research Council of Fundamental Science \& Technology funded by Ministry of Education, Science and Technology [Project: A Study on Satellite based Position Tracking Technology for Calamity Prevention and Public Safety Improvement] and Expert Education Program of Maritime Transportation Technology (GNSS Area), Ministry of Oceans and Fisheries of Korean government.

\section{REFERENCES}

Feng, Y., Wang, J. 2008, GPS RTK Performance Characteristics and Analysis, Journal of Global Positioning System, 7, 1-8

Hatch, R. R. 1982, The Synergism of GPS Code and Carrier Measurements, Proceedings of the Third International Geodetic Symposium on Satellite Dopler Positioning, II, 1213-1232

Hong, S., Lee, M. H., Kwon, S. K., \& Chun, H. H. 2004, Car Test for the Estimation of GPS/INS Alignment Errors, IEEE Trans Intell, Transport. System, 5, 208-218

Hong, S., Lee, M. H., Chun, H. H., Kwon, S. H., \& Speyer, J. L. 2005, Observability of error states in GPS/INS integration, IEEE Trans. Veh. Technol., 54, 731-743

Hwang, P. Y. C., \& Brown, R. G. 1990, GPS navigation: combining pseudorange with continuous carrier phase using a Kalman filter, Navigation: Journal of The Institute of Navigation, 37, 181-196

Hwang, P. Y. C., McGraw, G. A., \& Bader, J. R. 1999, Enhanced differential GPS carrier-smoothed-code processing using dual-frequency measurements, Journal of The 
Institute of Navigation, 26, 127-137

Kim, H. S. \& Lee, H. K. 2008, Position-Domain DD Hatch Filter to Maintain Float Solution Accuracy in RTK, Proceedings of International Symposium on GPS/GNSS 2008, 11-14

Lee, H. K. 2005, GAFAS: GNSSS Processing Software Modules for Accuracy and Safety, Report, APEC GNSS Implementation Team Meeting, Seoul

Lee, H. K. \& Rizos, C. 2008, Position-Domain Hatch Filter for Kinematic Differential GPS/GNSS, IEE Transactions on Aerospace and Electronics Systems, 44, 30-40

NovAtel Inc, 2010, OEMV Family Firmware Reference Manual, Revision 8, Calgary, Canada.

Parkinson, B. \& Axelad, P. 1996, Global Positioning System: Theory and Application, American Institute of Aeronautics and Astronautics

RTCM, 2006, RTCM Standard 10403.1 - Differential GNSS (Global Navigation Satellite Systems) Services, Version 3 Teunissen, P. J. G. 1994, A new method for fast carrier phase ambiguity estimation, Proceedings of IEEE Position, Location and Navigation Symposium, Las Vegas, Nevada, pp. 562-573

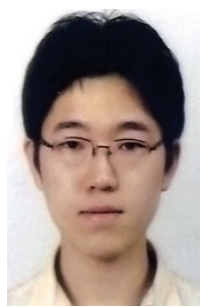

Hee Sung Kim received the M.S. degree in School of Electronics, Telecommunication \& Computer Engineering, Korea Aerospace University in 2009. $\mathrm{He}$ is a doctoral course student at Navigation and Information Systems Laboratory (NISL), Korea Aerospace University. His research interests include real-time GNSS Long-baseline, Network and L1 RTK and its applications.

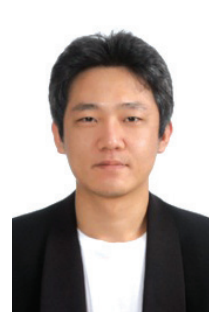

Kwang Ho Choi received the M.S. degree in School of Electronics, Telecommunication \& Computer Engineering, Korea Aerospace University. Since 2012, he is a doctoral course student at Navigation and Information Systems Laboratory (NISL), Korea Aerospace University. His research interests include network-based real-time high resolution TEC map generation and GPS/INS hybrid system applications.

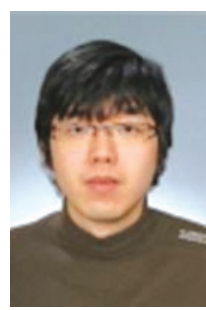

Je Young Lee received the M.S. degree in School of Electronics, Telecommunication \& Computer Engineering, Korea Aerospace University in 2011. $\mathrm{He}$ is a doctoral course student at Navigation and Information Systems Laboratory (NISL), Korea Aerospace University. His research interests include fault detection, adaptive filtering, INS and its applications.

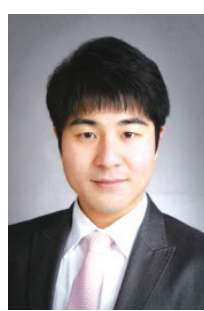

Joon Hoo Lim received the B.S. degree in Mechatronics Engineering, Korea Polytechnic University in 2009. He is a master course student at Navigation and Information Systems Laboratory (NISL), Korea Aerospace University. His research interests include hybrid positioning system, integrity and its applications.

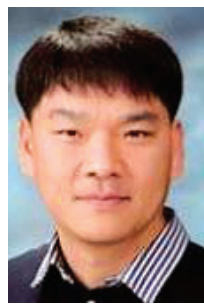

Se Bum Chun received his Ph.D. degree in Aerospace Engineering from Konkuk University in 2008 . He is a senior researcher in the Satellite Navigation team, CNS/ATM and Satellite Navigation Research Center in Korea Aerospace Research Institute (KARI). His research interests include GPS, INS, SLAM and non-linear system state estimation.

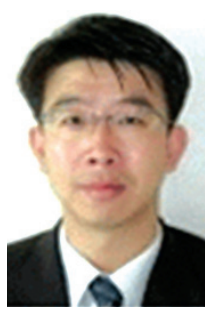

Hyung Keun Lee received the Ph.D. degree from the School of Electrical Engineering and Computer Science from Seoul National University, Korea in 2002. Since 2003, he has been with the School of Electronics, Telecommunication and Computer Engineering at Korea Aerospace University, Korea, as an Associate Professor. His research interests include positioning and navigation systems, sensor networks, and avionics. 